\title{
ON SPECTRA OF SECOND-ORDER DIFFERENTIAL OPERATORS ${ }^{(1)}$
}

\author{
BY \\ DANIEL RAY
}

1. Introduction. We propose to derive some results about the differential operator

$$
L \equiv \frac{1}{2} \Delta-V(x)
$$

on $N$-dimensional Euclidean space $R^{N}$ from a treatment based on the theory of random processes. These methods have been developed by Kac and others; most of the results of this paper have been suggested in Reference [1].

It has been shown by Rosenblatt [2] that for $V(x)$ a non-negative function satisfying a local Lipschitz condition at almost every point of $R^{N}$, the Green's function of the differential equation

$$
\frac{1}{2} \Delta \psi(x, t)-V(x) \psi(x, t)=\frac{\partial}{\partial t} \psi(x, t), \quad t>0,
$$

can be written in the explicit form

(3) $K(x, y ; t)=p(x-y, t) E\left\{\exp \left\{-\int_{0}^{t} V(x+x(\tau)) d \tau\right\} \mid x(t)=y-x\right\}$,

where

$$
p(x, t)=(2 \pi t)^{-N / 2} \exp \left\{-\frac{|x|^{2}}{2 t}\right\}
$$

and

$$
E\left\{\exp \left\{-\int_{0}^{t} V(x+x(\tau)) d \tau\right\} \mid x(t)=y-x\right\}
$$

denotes the average value of

$$
\exp \left\{-\int_{0}^{t} V(x+x(\tau)) d \tau\right\}
$$

Received by the editors July 10, 1953.

(1) The preparation of this paper was sponsored (in part) by the Office of Naval Research, USN. The paper comprises part of a thesis submitted under the direction of Professor Mark Kac to Cornell University. 
for all paths $x(\cdot)$ of a Wiener process on $R^{N}[5 ; 6]$ satisfying $x(0)=0, x(t)$ $=y-x$.

We shall generalize Rosenblatt's theorem, giving the explicit Green's functions of (2) and of the associated elliptic differential operator

$$
L_{s} \equiv \frac{1}{2} \Delta-(s+V(x))
$$

in certain open subsets $\Omega$ of $R^{N}$ with boundary values zero. It will be seen that a sufficient condition for the Green's functions to actually approach continuously the value zero at a boundary point $x_{0}$ of $\Omega$ is that there exist a conical sector with vertex at $x_{0}$, entirely outside $\Omega$. With a refinement of our methods, even weaker regularity conditions can be derived, although this seems the best that can be obtained with little effort.

The explicit Green's function will then be used to prove the following properties of the operator $L=L_{0}$ on the space $L^{2}(\bar{\Omega})$ of all functions with summable square on the closure $\bar{\Omega}$ of $\Omega$ :

A. If $\Omega$ is bounded, then the operator $L$, with boundary values zero, has a discrete spectrum of negative eigenvalues $\left\{-\lambda_{n}\right\}$, with eigenfunctions $\left\{\phi_{n}(x)\right\}$ forming a complete orthonormal basis in $L^{2}(\bar{\Omega})$ (Theorem III).

1. The eigenfunctions of $L$ have the asymptotic behavior

$$
\sum_{\lambda_{n}<\lambda} \phi_{n}^{2}(x) \sim\left(\frac{\lambda}{2 \pi}\right)^{N / 2} \frac{1}{\Gamma(N / 2+1)},
$$

as $\lambda \rightarrow \infty$ (Theorem IV).

2. The eigenvalues of $L$ have the asymptotic behavior

$$
N(\lambda)=\sum_{\lambda_{n}<\lambda} 1 \sim\left(\frac{\lambda}{2 \pi}\right)^{N / 2} \frac{m \bar{\Omega}}{\Gamma(N / 2+1)},
$$

as $\lambda \rightarrow \infty$, where $m \bar{\Omega}$ denotes the measure of the set $\bar{\Omega}$ (Theorem V).

B. If $\Omega$ is unbounded, and if $\lim _{|x| \rightarrow \infty, x \in \bar{\Omega}} V(x)=\infty$, then the operator $L$, with boundary values zero, has a discrete spectrum of negative eigenvalues $\left\{-\lambda_{n}\right\}$, with eigenfunctions $\left\{\phi_{n}(x)\right\}$ forming a complete orthonormal basis in $L^{2}(\bar{\Omega})$ (Theorem III).

1. The eigenfunctions of $L$ have the asymptotic behavior

$$
\sum_{\lambda_{n}<\lambda} \phi_{n}^{2}(x) \sim\left(\frac{\lambda}{2 \pi}\right)^{N / 2} \frac{1}{\Gamma(N / 2+1)},
$$

as $\lambda \rightarrow \infty$ (Theorem IV).

2. If in addition, for some $\delta>0$,

$$
m\{x \in \bar{\Omega} \mid V(x)<\lambda\} \sim m\left\{x \in \bar{\Omega} \mid \operatorname{Max}_{|y|<\delta, x+y \in \bar{\Omega}} V(x+y)<\lambda\right\} \sim \lambda^{\alpha} F(\lambda),
$$


with $\alpha \geqq 0$ and $F(\lambda)$ slowly increasing in the sense of Karamata, then the eigenvalues of $L$ have the asymptotic behavior

$N(\lambda)=\sum_{\lambda_{n}<\lambda} 1 \sim(2 \pi)^{-N} B(\lambda)=(2 \pi)^{-N} m\left\{x,\left.y\left|\frac{1}{2}\right| y\right|^{2}+V(x)<\lambda, x \in \bar{\Omega}\right\}$,

as $\lambda \rightarrow \infty$ (Theorem VI).

The results concerning the nature of the spectrum are well known $[3$, Chap. V]. The result concerning the asymptotic behavior of the eigenfunctions in both cases A and B is a generalization of Carleman's Theorem [1, p. 206]. The asymptotic relation for the eigenvalues in the case of bounded $\Omega$ is known as Weyl's Theorem; special cases are given in [9, Chap. VI]. These results have previously been obtained by the classical methods of operator theory, or of the theory of meromorphic functions; the proofs in the present paper are considerably simpler.

It is only with the asymptotic relation for the eigenvalues of $L$ when $\Omega$ is unbounded that a result hitherto unproven has been obtained by the methods of this paper. Titchmarsh [3, Chap. VI] has proved the following theorem, using the Sturmian theory and complex function theory:

Let $V(x)$, defined on the real line $R^{\prime}$, satisfy $\lim _{|x| \rightarrow \infty} V(x)=\infty$; suppose $V^{\prime \prime}(x)$ exists, and $V^{\prime \prime}(x) \geqq 0, \lim _{|x| \rightarrow \infty}\left|V^{\prime}(x)\right|=\infty, V^{\prime \prime}(x) \leqq\left|V^{\prime}(x)\right| r, 1<r$ $<4 / 3$, for $|x|$ sufficiently large. Then the operator $L \equiv(1 / 2) d^{2} / d x^{2}-V(x)$ on $L^{2}\left(R^{\prime}\right)$ has a spectrum of negative eigenvalues $\left\{-\lambda_{n}\right\}$ satisfying

$$
N(\lambda)=\sum_{\lambda_{n}<\lambda} 1=\frac{1}{2 \pi} B(\lambda)+O(1),
$$

as $\lambda \rightarrow \infty$, where

$$
B(\lambda)=m\left\{x, y \mid \frac{1}{2} y^{2}+V(x)<\lambda\right\}=2^{3 / 2} \int_{V(x)<\lambda}(\lambda-V(x))^{1 / 2} d x .
$$

Titchmarsh's result is stronger than ours where it applies, but even in the case $N=1$, our Theorem VI is true for potentials $V(x)$ which are not convex. Because of the use of Sturmian theory, Titchmarsh's proof cannot be generalized to dimension $N>1$, whereas the method of random process is almost unchanged for higher dimensions.

The result $N(\lambda) \sim(2 \pi)^{-N} B(\lambda)$ for dimension $N=3$ can be interpreted in the light of quantum theory by the transformation $y=\left(h / 2 \pi m^{1 / 2}\right) x, \lambda=E . L$ becomes the Schrödinger energy operator with potential $V_{1}(y)$ $=V\left(2 \pi\left(m^{1 / 2} / h\right) y\right)$;

$$
(2 \pi)^{-3} B(E)=\frac{2}{h} \int_{V_{1}(y)<E}\left(2 m\left(E-V_{1}(y)\right)\right)^{1 / 2} d y
$$

is the integral of the momentum of the system over a cycle in units of $h$. Hence 
$N(E) \sim(2 \pi)^{-3} B(E)$ expresses the equivalence at high energies of the BohrSommerfeld and Schrödinger theories. The result and the physicist's method of deriving it are usually known by the names of Wentzel, Kramers, and Brillouin.

We take for granted the reader's acquaintance with the theory of measure and integration connected with Wiener processes [5, Chap. VIII; 6, pp. 492$494]$. The theory deals with the construction of a probability measure on the space $C_{t}$ of possible paths of the process; that is, continuous functions $x(\tau)$, $0 \leqq \tau \leqq t$, with values in $R^{N}$, such that $x(0)=0$. The probability measure satisfies

$\operatorname{Prob}\left\{x\left(\tau_{j}\right) \in E_{j}, 1 \leqq j \leqq n\right\}=\int_{E_{1}} d x_{1} \cdots \int_{E_{n}} d x_{n} \prod_{1}^{n} p\left(x_{j}-x_{j-1}, \tau_{j}-\tau_{j-1}\right)$, where $x_{0}=\tau_{0}=0$, for Borel sets $E_{1}, \cdots, E_{n}$ in $R^{N}$ and times $0<\tau_{1}<\cdots$ $<\tau_{n} \leqq t$. There is likewise a conditional probability measure on the subset $C_{t, x}$ of $C_{t}$ of paths with $x(t)=x$, such that for any subset $F$ of $C_{t}$ in the Borel field generated by neighborhoods in $C_{t}$ of the above form, and for any Borel set $E$ in $R^{N}$,

$$
\int_{E} \operatorname{Prob}\left\{F \cap C_{t, x} \mid x(t)=x\right\} p(x, t) d x=\operatorname{Prob}\{F \cap\{x(t) \in E\}\} .
$$

We write integrals over $C_{t}$ or $C_{t, x}$ with respect to the Wiener measures as

$$
E\{\Phi(x(\cdot))\} \text { and } E\{\Phi(x(\cdot)) \mid x(t)=x\} \text {, }
$$

respectively; the integral over a subset $F$ of $C_{t}$ is denoted as $E\{\Phi(x(\cdot))$; $x(\cdot) \in F\}$.

We obtain the results listed under A and B by treating the Green's function $K(x, y ; t)$ of $(2)$ as the kernel of a semi-group of positive definite, selfadjoint, contraction operators on $L^{2}(\bar{\Omega})$. The infinitesimal generator of the semi-group is just the differential operator $L$, and hence the results concerning the nature of the spectrum of $L$ follow immediately from similar results for the semi-group. Using the explicit form for $K(x, y ; t)$, we shall show that the semigroup consists of completely continuous operators in the cases $\mathrm{A}$ and $\mathrm{B}$, and hence that the results hold which are stated there concerning the nature of the spectrum. The proof of complete continuity is a direct one, using a criterion of Tamarkin to show that the unit ball of $L^{2}(\bar{\Omega})$ is sent into a compact set by each of the operators. Hence we have a direct and explicit proof that the operator $L$ has a discrete spectrum in the cases A and B.

Finally we prove the results concerning the asymptotic behavior of the eigenfunctions and eigenvalues of $L$. From the previous sections we find that

$$
\sum_{n} \exp \left\{-\lambda_{n} t\right\} \phi_{n}^{2}(x)=K(x, x ; t), \quad \sum_{n} \exp \left\{-\lambda_{n} t\right\}=\int_{\bar{\Omega}} K(x, x ; t) d x
$$


We next infer from (3) that as $t \rightarrow 0, K(x, x ; t) \sim(2 \pi t)^{-N / 2}$; a Tauberian argument therefore implies

$$
\sum_{\lambda_{n}<\lambda} \phi_{n}^{2}(x) \sim\left(\frac{\lambda}{2 \pi}\right)^{N / 2} \frac{1}{\Gamma(N / 2+1)},
$$

as $\lambda \rightarrow \infty$. If $\Omega$ is bounded, it is fairly easy to see that the asymptotic relation can be integrated over $\bar{\Omega}$ with respect to $x$, yielding

$$
N(\lambda)=\sum_{\lambda_{n}<\lambda} 1 \sim\left(\frac{\lambda}{2 \pi}\right)^{N / 2} \frac{m \bar{\Omega}}{\Gamma(N / 2+1)},
$$

as $\lambda \rightarrow \infty$.

If $\Omega$ is unbounded, a Tauberian theorem can still be used to derive the asymptotic formula for $N(\lambda)$ only if additional hypotheses are assumed concerning the potential $V(x)$. If $m\{x \in \bar{\Omega} \mid V(x)<\lambda\} \sim \lambda^{\alpha} F(\lambda)$, with $\alpha \geqq 0$ and $F(\lambda)$ slowly increasing, then the formula can be obtained from a Tauberian theorem of Karamata from the relation

$$
\int_{\bar{\Omega}} K(x, x ; t) d x \sim \frac{1}{(2 \pi t)^{N / 2}} \int_{\bar{\Omega}} e^{-t V(x)} d x, \quad t \rightarrow 0 .
$$

To prove this last equation, however, we require in addition a condition on the smoothness of $V(x)$.

These restrictions are actually necessary for reasons more inherent in the nature of the problem. This is illustrated by the potential $V(x)=\log ^{+}|x|$ on $R^{N}$, for which none of the above conditions hold. It can still be shown, however, that in this case,

$$
\int_{0}^{\lambda} e^{-\lambda N} d N(\lambda)=\sum_{\lambda_{n}<\lambda} e^{-N \lambda} x 2 \lambda /(2 N)^{N / 2} \Gamma\left(\frac{N}{2}\right) .
$$

It seems likely that no closer approximation to $N(\lambda)$ can be found from only the asymptotic behavior of $V(x)$, since a great deal of variation can be allowed in $N(\lambda)$ without appreciably changing the behavior of its Laplace transform.

We believe that the association of probability theory with the differential equation (2) is quite natural, and that the form (3) of the Green's function makes intuitively obvious many of the deeper properties of the differential operator $L$. It is hoped that the technique will be applicable to other problems, in particular to investigate the operator $L$ in the case of a continuous spectrum.

2. Green's functions associated with the operator $L$. The following theorem forms the basis of our treatment of the differential operator $L$. The proof is only a slight modification of that of Rosenblatt [2], and so only an outline of it is given here. 
TheOREM I. Let $\Omega$ be an open set in $R^{N}$ such that at each boundary point $x$ of $\Omega$, there is a sphere with center $x$, some open sector of which is entirely outside the closure $\bar{\Omega}$ of $\Omega$.

Let $V(x)$ be a non-negative Borel measurable function defined on $\bar{\Omega}$, bounded on each bounded subset of $\bar{\Omega}$. Let $V(x)$ satisfy, at almost every point $x$ in $\bar{\Omega}, a$ Lipschitz condition of the form

$$
\left|V\left(x^{\prime}\right)-V(x)\right|<M(x)\left|x-x^{\prime}\right|^{\alpha}, \quad 0<\alpha \leqq 1,
$$

$x^{\prime}$ in some neighborhood of $x$.

For $x, y$ in $\bar{\Omega}, t>0, s>0$, set

$K(x, y ; t)=p(x-y, t)$

$$
\begin{gathered}
\cdot E\left\{\exp \left\{-\int_{0}^{t} V(y+x(\tau)) d \tau\right\} ; y+x(\tau) \in \bar{\Omega}, 0 \leqq \tau \leqq t \mid x(t)=x-y\right\}, \\
H(x, y ; s)=\int_{0}^{\infty} e^{-s t} K(x, y ; t) d t .
\end{gathered}
$$

Then

(1) $K(x, y ; t)$ is the Green's function of the differential equation (2)

$$
\frac{1}{2} \Delta \phi(x, t)-V(x) \phi(x, t)=\frac{\partial}{\partial t} \phi(x, t), \quad x \in \Omega, t>0,
$$

with boundary values zero: If $\psi(x)$ is a continuous function in $L^{2}(\bar{\Omega})$, then

$$
\phi(x, t)=\int_{\bar{\Omega}} K(x, y ; t) \psi(y) d y
$$

is the unique function with the properties:

$\Delta \phi(x, t)$ and $\partial \phi(x, t) / \partial t$ exist at each point $x$ in $\Omega$ at which the Lipschitz condition (5) holds, and for each $t>0$, and $2^{-1} \Delta \phi(x, t)-\partial \phi(x, t) / \partial t=V(x) \phi(x, t)$; $\phi(x, t)$ is continuous on $\bar{\Omega}$ for each $t>0$, and $\phi(x, t)=0$ on the boundary of $\Omega$;

$$
\lim _{t \rightarrow 0} \phi(x, t)=\psi(x) \text { for each } x \text { in } \Omega \text {; }
$$$$
\phi(x, t) \text { is in } L^{2}(\bar{\Omega}) \text { for each } t>0 \text {, and } \lim _{t \rightarrow 0}\|\phi(x, t)-\psi(x)\|=0,
$$

(2) $H(x, y ; s)$ is the Green's function of the differential operator

$$
L_{s} \phi(x)=1 / 2 \Delta \phi(x)-(s+V(x)) \phi(x),
$$

on $\Omega$ with boundary values zero: If $\psi(x)$ is a continuous function in $L^{2}(\bar{\Omega})$, then

$$
\phi(x)=\int_{\bar{\Omega}} H(x, y ; s) \psi(y) d y
$$


is the unique function with the properties:

$\Delta \phi(x)$ exists at each point $x$ in $\Omega$ at which the Lipschitz condition (5) holds, and $2^{-1} \Delta \phi(x)=(s+V(x)) \phi(x)-\psi(x)$;

$\phi(x)$ is continuous on $\bar{\Omega}$ and $\phi(x)=0$ on the boundary of $\Omega$;

$\phi(x)$ is in $L^{2}(\bar{\Omega})$.

If $\Omega=R^{N}$ and $V(x)$ is bounded on $R^{N}$, besides satisfying the hypotheses of the theorem, then Rosenblatt has proved that $K(x, y ; t)$ has second spatial derivatives and a first time derivative, satisfying (2), at each point $x$ in $R^{N}$ at which the Lipschitz condition holds for $V(x)$, and at each $t>0$. This same result can be extended to the general case by the following methods:

Set $V_{n}(x)=V(x)$ if $V(x) \leqq n$ and $\bar{x}$ is in $\Omega ; V_{n}(x)=n$ if $V(x)>n$ and $x \in \bar{\Omega}$ or if $x \in \Omega$. Set

$$
K_{n}(x, y ; t)=p(x-y, t) E\left\{\exp \left\{-\int_{0}^{t} V_{n}(y+x(\tau) d \tau\} \mid x(t)=x-y\right\} .\right.
$$

Then $K_{n}(x, y ; t) \geqq K_{n+1}(x, y ; t)$, and

$\lim _{n \rightarrow \infty} K_{n}(x, y ; t)=K(x, y ; t)=p(x-y, t)$

. $E\left\{\exp \left\{-\int_{0}^{t} V(y+x(\tau)) d \tau\right\} ; y+x(\tau) \in \bar{\Omega}, 0 \leqq \tau \leqq t \mid x(t)=x-y\right\}$.

The latter statement is proved if we show that for every path $x(\cdot)$ of the Wiener process such that $x(t)=x-y$,

$$
\lim _{n \rightarrow \infty} \exp \left\{-\int_{0}^{t} V_{n}(y+x(\tau)) d \tau\right\}=\exp \left\{-\int_{0}^{t} V(y+x(\tau)) d \tau\right\} C_{\bar{\Omega}}(x(\cdot)),
$$

where $C_{\bar{\Omega}}(x(\cdot))=1$ if $x(\tau)+y \in \bar{\Omega}$ for $0 \leqq \tau \leqq t$, and $C_{\bar{\Omega}}(x(\cdot))=0$ otherwise. But if the path $x(\cdot)$ satisfies $x(\tau)+y \in \bar{\Omega}, 0 \leqq \tau \leqq t$, then certainly

$$
\exp \left\{-\int_{0}^{t} V_{n}(y+x(\tau)) d \tau\right\} \rightarrow \exp \left\{-\int_{0}^{t} V(y+x(\tau)) d \tau\right\} .
$$

If on the other hand $y+x\left(\tau_{0}\right)$ is in the complement of $\bar{\Omega}$ for some $\tau_{0}, 0 \leqq \tau_{0} \leqq t$, then since the Wiener path $x(\cdot)$ is continuous, $x(\tau)+y$ is in the complement of $\bar{\Omega}$ for all $\tau$ in some interval of time of length $\delta>0$. Hence

$$
\lim _{n \rightarrow \infty} \exp \left\{-\int_{0}^{t} V_{n}(x+x(\tau)) d \tau\right\} \leqq \lim _{n \rightarrow \infty} e^{-n \delta}=0 .
$$

Let $x_{0}$ be a point in $\Omega$ at which the Lipschitz condition on $V(x)$ holds, and take $t>0$. Since $\Omega$ is open, there is a sphere of radius say $r_{0}$ about $x_{0}$, entirely in $\Omega$. Let $\rho(r)$ be a function of the real variable $r \geqq 0$, sufficiently smooth, such that $\rho(r)=1$ for $0 \leqq r \leqq r_{0} / 4, \rho(r)=0$ for $r \geqq r_{0} / 2$. Set $q\left(\xi, t^{\prime}\right)=p\left(\xi, t^{\prime}\right) \rho(|\xi|)$.

By Rosenblatt's proof, 


$$
\frac{1}{2} \Delta K_{n}(x, y ; t)-V_{n}(x) K_{n}(x, y ; t)=\frac{\partial}{\partial t} K_{n}(x, y ; t)
$$

at every point of $R^{N}$ at which (5) holds. Green's formula can be applied, since $\left(2^{-1} \Delta-\partial / \partial t\right) K_{n}$ is essentially bounded in the sphere $\left|x-x_{0}\right| \leqq r_{0}$, and with little difficulty we obtain for $\left|x-x_{0}\right| \leqq r_{0} / 2$

$$
\begin{aligned}
& \int_{0}^{t} d t^{\prime} \int_{R^{N}} d \xi\left(\frac{1}{2} \Delta_{\xi}+\frac{\partial}{\partial t^{\prime}}\right) q\left(x-\xi, t-t^{\prime}\right) K_{n}\left(\xi, y ; t^{\prime}\right) \\
& =K_{n}(x, y ; t)-q(x-y, t)+\int_{0}^{t} d t^{\prime} \int_{R^{N}} d \xi q\left(x-\xi, t-t^{\prime}\right) V_{n}(\xi) K_{n}\left(\xi, y ; t^{\prime}\right) .
\end{aligned}
$$

Now since each of the integrands vanishes outside the sphere $\left|\xi-x_{0}\right| \leqq r_{0}$, and since in the sphere $V(\xi)$ is bounded, we may let $n$ approach infinity, obtaining the following integral equation for $K(x, y ; t)$ :

$$
\begin{aligned}
& K(x, y ; t)+\int_{0}^{t} d t^{\prime} \int_{\bar{\Omega}} d \xi q\left(x-\xi, t-t^{\prime}\right) V(\xi) K\left(\xi, y ; t^{\prime}\right) \\
& \quad=q(x-y, t)+\int_{0}^{t} d t^{\prime} \int_{\bar{\Omega}} d \xi\left(\frac{1}{2} \Delta_{\xi}+\frac{\partial}{\partial t^{\prime}}\right) q\left(x-\xi, t-t^{\prime}\right) \cdot K\left(\xi, y ; t^{\prime}\right) .
\end{aligned}
$$

As in Rosenblatt's proof, it now follows that $\Delta K(x, y ; t)$ and $\partial K(x, y ; t) / \partial t$ exist whenever (5) holds at $x \in \Omega$, and $t>0$. Moreover, these derivatives can be calculated $[4$, pp. 189,193$]$, and it is found that

$$
\begin{aligned}
\frac{1}{2} \Delta K\left(x_{0}, y ; t\right) & -\frac{\partial}{\partial t} K\left(x_{0}, y ; t\right) \\
= & V\left(x_{0}\right) K\left(x_{0}, y ; t\right)+\left(\frac{1}{2} \Delta-\frac{\partial}{\partial t}\right) q\left(x_{0}-\xi, t\right) \\
& -\int_{0}^{t} d t^{\prime} \int_{\bar{\Omega}} d \xi\left(\frac{1}{2} \Delta_{\xi}+\frac{\partial}{\partial t^{\prime}}\right) q\left(x_{0}-\xi, t-t^{\prime}\right) V(\xi) K\left(\xi, y ; t^{\prime}\right) \\
& +\int_{0}^{t} d t^{\prime} \int_{\bar{\Omega}} d \xi\left(\frac{1}{2} \Delta_{\xi}+\frac{\partial}{\partial t^{\prime}}\right)^{2} q\left(x_{0}-\xi, t-t^{\prime}\right) K\left(\xi, y ; t^{\prime}\right) \\
= & \lim _{n \rightarrow \infty}\left\{V_{n}\left(x_{0}\right) K_{n}\left(x_{0}, y ; t\right)+\left(\frac{1}{2} \Delta-\frac{\partial}{\partial t}\right) q\left(x_{0}-\xi, t\right)\right. \\
& -\int_{0}^{t} d t^{\prime} \int_{\bar{\Omega}} d \xi\left(\frac{1}{2} \Delta_{\xi}+\frac{\partial}{\partial t^{\prime}}\right) q\left(x_{0}-\xi, t-t^{\prime}\right) V_{n}(\xi) K_{n}\left(\xi, y ; t^{\prime}\right) \\
& \left.+\int_{0}^{t} d t^{\prime} \int_{\Omega} d \xi\left(\frac{1}{2} \Delta_{\xi}+\frac{\partial}{\partial t^{\prime}}\right)^{2} q\left(x_{0}-\xi, t-t^{\prime}\right) K_{n}(\xi, y ; t)\right\}
\end{aligned}
$$




$$
\begin{aligned}
& =\lim _{n \rightarrow \infty}\left\{\frac{1}{2} \Delta K_{n}\left(x_{0}, y ; t\right)-\frac{\partial}{\partial t} K_{n}\left(x_{0}, y ; t\right)\right\} \\
& =\lim _{n \rightarrow \infty}\left\{V_{n}\left(x_{0}\right) K_{n}\left(x_{0}, y ; t\right)\right\}=V\left(x_{0}\right) K\left(x_{0} y ; t\right) .
\end{aligned}
$$

That for every continuous function $\psi(x)$ in $L^{2}(\bar{\Omega})$

$$
\phi(x, t)=\int_{\Omega} K(x, y ; t) \psi(y) d y
$$

has second spacial derivatives and a single time derivative satisfying (2) whenever $x$ is a point at which (5) holds and whenever $t>0$ can now be proved by substituting the equation ( 7$)$ for $K(x, y ; t)$, obtaining an integral equation for $\phi(x, t)$, to which the same analysis as above applies. The initial value properties for $\phi(x, t)$ follow from the relation

$$
\begin{aligned}
& \phi(x, t)= \int_{\bar{\Omega}} K(x, y ; t) \psi(y) d y \\
&= \int_{\bar{\Omega}} E\left\{\exp \left\{-\int_{0}^{t} V(y+x(\tau)) d \tau\right\} ;\right. \\
&\quad y+x(\tau) \in \bar{\Omega}, 0 \leqq \tau \leqq t \mid x(t)=x-y\} \cdot \psi(y) p(x-y, t) d y \\
&= E\left\{\exp \left\{-\int_{0}^{t} V(x+x(\tau)) d \tau\right\} \psi(x+x(t)) ;\right. \\
&x+x(\tau) \in \bar{\Omega}, 0 \leqq \tau \leqq t\},
\end{aligned}
$$

which is easily derived from equation (4). From (8) it is also clear that $\phi(x, t)$ is in $L^{2}(\bar{\Omega})$ for all $t>0$, and $\|\phi(x, t)\| \leqq\|\psi\|$.

It can be shown [4] that $\Delta \phi(x, t)$ has the bound $K t^{-1 / 2}$ for $x$ in a small neighborhood of a point $x_{0}$ of $\Omega$ at which (5) holds. Hence

$$
\phi(x)=\int_{0}^{\infty} e^{-s t} \phi(x, t) d t=\int_{\bar{\Omega}} H(x, y ; s) \psi(y) d y
$$

has second derivatives at $x_{0}$, and from the initial value relations for $\phi(x, t)$, these satisfy

$$
\frac{1}{2} \Delta \phi\left(x_{0}\right)-\left(s+V\left(x_{0}\right)\right) \phi\left(x_{0}\right)=-\psi\left(x_{0}\right) .
$$

It is clear also that $\phi(x)$ is in $L^{2}(\bar{\Omega})$.

The uniqueness of the solutions $\phi(x, t)$ and $\phi(x)$ is proved by standard methods. If the boundary of $\Omega$ is sufficiently smooth, the uniqueness follows 
from Green's formula. In general, using the result for $\Omega$ with smooth boundaries, it can be shown that a solution of (2) can have no positive maximum or negative minimum, from which the uniqueness follows.

It remains to show that $\phi(x)$ and $\phi(x, t)$ have the proper boundary values. For this it is sufficient to show that $\lim _{x \rightarrow x_{0}, x \in \Omega} K(x, y ; t)=0$ if $y \in \Omega, t>0$, and if $x_{0}$ is a boundary point of $\Omega$. To this purpose, we give the following result in the theory of Wiener processes, due to G. A. Hunt.

Lemma 1. Let $C$ be the open sector in $R^{N}$ :

$$
C=\left\{x=\left(x^{1}, \cdots, x^{N}\right)\left|\left(x^{2}\right)^{2}+\cdots+\left(x^{N}\right)^{2}<b^{2}\left(x^{1}\right)^{2},\right| x \mid<a\right\} .
$$

Let $\left\{t_{n}\right\}$ be a sequence of times such that $0 \leqq t_{n+1} \leqq t_{n} \leqq 1$ and $t_{n} \rightarrow 0$. Then

Prob $\left\{x\left(t_{n}\right) \in C\right.$ for infinitely many $\left.n\right\}=1$.

Set $X_{n}=x\left(t_{n}\right)-x\left(t_{n+1}\right)$. Then for $n_{0}=1,2, \cdots,\left\{X_{1}, \cdots, X_{n_{0}}\right\}$ are independent of $\left\{\sum_{n}^{\infty} X_{k}, n>n_{0}\right\}$, so that the event $\left\{x\left(t_{n}\right) \in C\right.$ for infinitely many $\left.n>n_{0}\right\}=\left\{\sum_{n}^{\infty} X_{k} \in C\right.$ for infinitely many $\left.n>n_{0}\right\}$ is independent of $\left\{X_{1}, \cdots, X_{n_{0}}\right\}$. Hence, by the zero-one law of probability [8, p. 69], Prob $\left\{x\left(t_{n}\right) \in C\right.$ for infinitely many $\left.n\right\}$ is zero or one. But

$1-\operatorname{Prob}\left\{x\left(t_{n}\right) \in C\right.$ for infinitely many $\left.n\right\}$

$$
\begin{aligned}
& =\lim _{n_{0} \rightarrow \infty} \operatorname{Prob}\left\{x\left(t_{n}\right) \notin C, \text { for all } n \geqq n_{0}\right\} \\
& \leqq \lim _{n_{0} \rightarrow \infty} \operatorname{Prob}\left\{x\left(t_{n_{0}}\right) \notin C\right\} \\
& \leqq \lim _{n_{0} \rightarrow \infty}\left[1-w_{c} \int_{|y|<a} p\left(y, t_{n_{0}}\right) d y\right] \\
& =1-w_{c}
\end{aligned}
$$

where $w_{c}$ is the ratio of the solid angle subtended by the sector $C$ to the total solid angle $2 \pi^{N / 2} / \Gamma(N / 2)$ in $R^{N}$. Hence Prob $\left\{x\left(t_{n}\right) \in C\right.$ for infinitely many $\left.n\right\}$ $\geqq w_{c}>0$, so that by the zero-one law, Prob $\left\{x\left(t_{n}\right) \in C\right.$ for infinitely many $\left.n\right\}$ $=1$.

We now prove $\lim _{x \rightarrow x_{0}, x \in \Omega} K(x, y ; t)=0$, whenever $x_{0}$ is a boundary point of $\Omega$. Certainly

$$
\lim _{x \rightarrow x_{0}, x \in \Omega} K(x, y ; t) \leqq \lim _{x \rightarrow x_{0}, x \in \Omega} K_{n}(x, y ; t)=K_{n}\left(x_{0} ; y ; t\right),
$$

for every positive integer $n$, so that

$$
\begin{aligned}
& \lim _{x \rightarrow x_{0}, x \in \Omega} K(x, y ; t) \leqq \lim _{n \rightarrow \infty} K_{n}\left(x_{0}, y ; t\right)=K\left(x_{0}, y ; t\right) \\
& \leqq p\left(x_{0}-y, t\right) \text { Prob }\left\{x_{0}+x(\tau) \in \bar{\Omega}, 0 \leqq \tau \leqq t \mid x(t)=y-x_{0}\right\} .
\end{aligned}
$$

By the regularity hypothesis on the set $\Omega$, there is an open conical sector $C$ 
with vertex $x_{0}$, entirely outside $\bar{\Omega}$. Let $C_{1}$ be the translation of $C$ by $-x_{0}$ in $R^{N}$. Then

$\lim _{x \rightarrow x_{0}, x \in \Omega} K(x, y ; t) \leqq p\left(x_{0}-y, t\right)$ Prob $\left\{x(\tau) \notin C_{1}, 0 \leqq \tau \leqq t \mid x(t)=y\right\}$

$$
\begin{aligned}
& \leqq p\left(x_{0}-y, t\right) \operatorname{Prob}\left\{x(\tau) \notin C_{1}, 0 \leqq \tau \leqq \frac{t}{2} \mid x(t)=y\right\} \\
& =\frac{p\left(x_{0}-y, t\right)}{p(y, t)} E\left\{p\left(x-y\left(\frac{t}{2}\right), t-\frac{t}{2}\right) ; x(\tau) \notin C_{1} ; 0 \leqq \tau \leqq \frac{t}{2}\right\} \\
& \leqq(\pi t)^{N / 2} \frac{p\left(x_{0}-y, t\right)}{p(y, t)} \operatorname{Prob}\left\{x(\tau) \notin C_{1}, 0 \leqq \tau \leqq \frac{t}{2}\right\}
\end{aligned}
$$$$
=0
$$

by Lemma 5 .

Since $K(x, y ; t) \geqq 0$, we have shown that $K(x, y ; t)$ is continuous on $\bar{\Omega}$, with value zero on the boundary of $\bar{\Omega}$. The same properties therefore hold for $\phi(x, t)$ and $\phi(x)$, and the proof of Theorem I is complete.

TheOREM II. Let $\Omega$ and $V(x)$ be as in Theorem $\mathrm{I}$; then $K(x, y ; t)$, defined in equation (6) is the kernel of a semi-group of operators

$$
\psi(x) \rightarrow K_{t} \psi(x)=\int_{\bar{\Omega}} K(x, y ; t) \psi(y) d y, \quad t>0,
$$

on the Hilbert space $L^{2}(\bar{\Omega})$. Each operator $K_{t}$ is self-adjoint, positive definite, and is a strict contraction: $\left\|K_{t} \psi\right\|<\|\psi\|$ if $\psi \neq 0$. The semi-group is strongly continuous for $t \geqq 0$ : that is, $\lim _{\Delta t \rightarrow 0}\left\|K_{t+\Delta t} \psi-K_{t} \psi\right\|=0$ for every $\psi$ in $L^{2}(\bar{\Omega})$ and for $t \geqq 0$, where $K_{0} \psi=\psi$.

We omit the proof of Theorem II. It follows either from the fact that $K(x, y ; t)$ is the Green's function of (2), or directly from the theory of Wiener integrals, using equation (8). In the latter method of proof, the only step which is not trivial is the proof of the semi-group property $K_{t+t^{\prime}}=K_{t} K_{t^{\prime}}$.

3. Nature of the spectrum of $L=2^{-1} \Delta-V(x)$. We are now prepared to prove from Theorems I and II the results stated in the introduction under A and B. First we prove that if $\Omega$ is bounded or if $\lim _{|x| \rightarrow \infty, x \in \Omega} V(x)=\infty$, then $L$ has a discrete spectrum. This is a simple corollary of the fact that the operators $K_{t}$ of Theorem II are completely continuous in these cases. This in turn can be quite directly derived from equation (8), using the following calculation of the measure of certain subsets of the Wiener spaces $C_{t, x}$ and $C_{t}$.

Lemma 2. If $a>0$, then

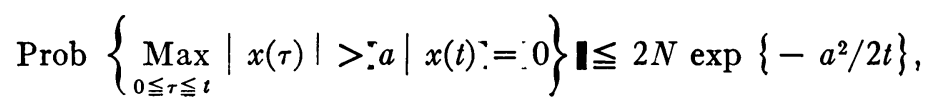




$$
\operatorname{Prob}\left\{\operatorname{Max}_{0 \leqq \tau \leqq t}|x(\tau)|>a\right\} \leqq 3 \int_{|\boldsymbol{y}|>a / 4} p(y, t) d y .
$$

Let $I a$ be the cube in $R^{N}\left\{x=\left(x^{\prime}, \cdots, x^{N}\right)|| x^{i} \mid \leqq a, 1 \leqq i \leqq N\right\}$. Then by Theorem $\mathrm{I}$, if $x$ is in $I a$,

$$
p(x, t) \operatorname{Prob}\{x(\tau) \in I a, 0 \leqq \tau \leqq t \mid x(t)=x\}=K(x, 0 ; t),
$$

where $K(x, y ; t)$ is the Green's function of $2^{-1} \Delta \phi=\partial \phi / \partial t$ with boundary values zero on the boundary of $I a$. It is easily verified that

$$
K(x, 0 ; t)=\frac{1}{a^{N}} \prod_{i=1}^{N} \sum_{n=1}^{\infty} \cos \frac{\pi}{a}\left(n+\frac{1}{2}\right) x^{i} \exp \left\{-\frac{\pi^{2}}{2 a^{2}}\left(n+\frac{1}{2}\right)^{2} t\right\} .
$$

Applying the Poisson summation formula,

$$
\begin{gathered}
\frac{1}{a} \sum_{n=0}^{\infty} \cos \frac{\pi}{a}\left(n+\frac{1}{2}\right) x^{i} \exp \left\{-\frac{\pi^{2}}{2 a^{2}}\left(n+\frac{1}{2}\right)^{2} t\right\} \\
=\frac{\exp \left\{-\left(x^{i}\right)^{2} / 2 t\right\}}{(2 \pi t)^{1 / 2}}\left(1-\sum_{1}^{\infty}(-1)^{k-1}\left(\exp \left\{-\frac{2 a k}{t}\left(a k-x^{i}\right)\right\}\right.\right. \\
\left.\left.\quad+\exp \left\{-\frac{2 a k}{t}\left(a k+x^{i}\right)\right\}\right)\right) \\
\geqq \frac{\exp \left\{-\left(x^{i}\right)^{2} / 2 t\right\}}{(2 \pi t)^{1 / 2}}\left(1-2 \exp \left\{-\frac{2 a}{t}\left(a-\left|x^{i}\right|\right)\right\}\right),
\end{gathered}
$$

since the terms of the alternating series decrease for $\left|x^{i}\right|<a$. Hence

$$
\begin{aligned}
\operatorname{Prob}\left\{\operatorname{Max}_{0 \leqq \tau \leqq t}|x(\tau)| \leqq a \mid x(t)\right. & =0\} \\
& \geqq \operatorname{Prob}\{x(\tau) \in I a / 2,0 \leqq \tau \leqq t \mid x(t)=0\} \\
& \geqq \frac{1}{p(0, t)} \prod_{i=1}^{N} \frac{1}{(2 \pi t)^{1 / 2}}\left(1-2 \exp \left\{-\frac{a^{2}}{2 t}\right\}\right) \\
& \geqq 1-2 N \exp \left\{-\frac{a^{2}}{2 t}\right\},
\end{aligned}
$$

and this is equivalent to equation (9).

To derive equation (10), we make use of the relation (4) satisfied by the conditional probability measure:

$\operatorname{Prob}\{x(\tau) \in I a, 0 \leqq \tau \leqq t\}$

$$
=\int_{R^{N}} p(x, t) \text { Prob }\{x(\tau) \in I a, 0 \leqq \tau \leqq t \mid x(t)=x\} d x
$$




$$
\begin{aligned}
& \geqq \int_{I a}\left(1-2 \sum_{1}^{N} \exp \left\{-\frac{2 a}{t}\left(a-\left|x^{i}\right|\right)\right\}\right) p(x, t) d x \\
& =1-\int_{R^{N}-I a} p(x, t) d x-2 \sum_{1}^{N}\left(\int_{-a}^{a} \frac{\exp \left\{x^{2} / 2 t\right\}}{(2 \pi t)^{1 / 2}} d x\right)^{N-1} \\
& \cdot 2 \int_{a}^{2 a} \frac{\exp \left\{-x^{2} / 2 t\right\}}{(2 \pi t)^{1 / 2}} d x \\
& \geqq 1-3 \int_{R^{N}-I a} p(x, t) d x .
\end{aligned}
$$

Therefore

$$
\begin{aligned}
\operatorname{Prob}\left\{\operatorname{Max}_{0 \leqq \tau \leqq t}|x(\tau)| \leqq a\right\} \geqq \operatorname{Prob}\{x(\tau) \in I a / 2,0 \leqq \tau \leqq t\} \\
\geqq 1-3 \int_{R^{N}-I a / 2} p(x, t) d x \geqq 1-3 \int_{|y|>a / 4} p(y, t) d y,
\end{aligned}
$$

which yields equation (10).

We immediately use this result to prove the complete continuity of each operator $K_{t}$ in the semi-group under the conditions described in A and B of the introduction.

Lemma 3. Let $\Omega$ and $V(x)$ be as in Theorem $\mathrm{I}$, and suppose also either that $\Omega$ is bounded or else that $\lim _{|x|+\infty, x \in \Omega} V(x)=\infty$. Then for each $t>0, K_{t}$ :

$$
K_{t} \psi(x)=\int_{\bar{\Omega}} K(x, y ; t) \psi(y) d y
$$

is completely continuous on $L^{2}(\bar{\Omega})$. Hence $K_{t}$ has a countable set of eigenvalues $\exp \left(-\lambda_{j} t\right\}$, with eigenfunctions $\left\{\phi_{j}(x)\right\}$ independent of $t$ and forming a complete orthonormal basis in $L^{2}(\bar{\Omega})$.

The complete continuity of $K_{t}, t>0$, is a consequence of a theorem of Tamarkin [7], if we prove

(1) $K_{t} \psi(x)$ is bounded uniformly for $\psi$ in $L^{2}(\bar{\Omega})$ with $\|\psi\|=1$ and for $x$ in $\bar{\Omega}$.

(2) $\int_{|x|>a}\left|K_{t} \psi(x)\right|^{2} d x \rightarrow 0$ as $a \rightarrow \infty$, uniformly for $\psi$ in $L^{2}(\bar{\Omega})$ with $\|\psi\|=1$.

But (1) is easy, using equation (8):

$$
\begin{aligned}
\left|K_{t} \psi(x)\right| \leqq E\left\{\exp \left\{-\int_{0}^{t} V(x+x(\tau)) d \tau\right\}|\psi(x+x(t))| ;\right. \\
x+x(\tau) \in \bar{\Omega}, 0 \leqq \tau \leqq t\} \\
\leqq E\{|\psi(x+x(t))| ; x+x(\tau) \in \bar{\Omega}, 0 \leqq \tau \leqq t\}
\end{aligned}
$$




$$
\begin{aligned}
& \leqq \int_{\bar{\Omega}} p(y-x, t)|\psi(y)| d y \leqq\left[\int_{R^{N}}(p(y, t))^{2} d y\right]^{1 / 2}\|\psi\| \\
& =(4 \pi t)^{-N / 2}
\end{aligned}
$$

if $\|\psi\|=1$. Moreover the statement (2) is trivial if $\Omega$ is bounded, so that in this case Tamarkin's criterion certainly holds.

To prove (2) in general, note first that by hypothesis exp $\{-t V(x)\}$ $<\delta(a)$ if $|x|>a / 2, x \in \Omega$, where $\delta(a) \rightarrow 0$ as $a \rightarrow \infty$. Hence if $x \in \Omega$ and $|x|>a$,

$$
\begin{aligned}
E\{\exp \{ & \left.\left.-2 \int_{0}^{t} V(x+x(\tau)) d \tau\right\} ; x+x(\tau) \in \bar{\Omega}, 0 \leqq \tau \leqq t\right\} \\
\leqq & \operatorname{Prob}\{x+x(\tau) \in \bar{\Omega}, 0 \leqq \tau \leqq t ; \underset{0 \leqq \tau \leqq t}{\operatorname{Max}}|x(\tau)|>a / 2\} \\
& +(\delta(a))^{2} \operatorname{Prob}\left\{x+x(\tau) \in \bar{\Omega}, 0 \leqq \tau \leqq t ; \operatorname{Max}_{0 \leqq \tau \leqq t}|x(\tau)| \leqq \frac{a}{2}\right\} \\
\leqq & 3 \int_{|y|>a / 8} p(y, t) d y+(\delta(a))^{2} .
\end{aligned}
$$

Thus

$$
\begin{aligned}
& \int_{|x|>a}\left|K_{t} \psi(x)\right|^{2} d x \\
& \leqq \int_{|x|>a} d x E\left\{\exp \left\{-2 \int_{0}^{t} V(x+x(\tau)) d \tau\right\} ; x+x(\tau) \in \bar{\Omega}, 0 \leqq \tau \leqq t\right\} \\
& \quad \cdot E\left\{|\psi(x+x(t))|^{2} ; x+x(\tau) \in \bar{\Omega}, 0 \leqq \tau \leqq t\right\}
\end{aligned}
$$

by Schwarz's inequality applied to (8),

$$
\begin{aligned}
& \leqq\left(3 \int_{|x|>a / 8} p(x, t) d x+(\delta(a))^{2}\right) \\
& \cdot \int_{|x|>a} d x E\left\{|\psi(x+x(t))|^{2} ; x+x(\tau) \in \bar{\Omega}, 0 \leqq \tau \leqq t\right\} \\
& \leqq\left(3 \int_{|x|>a / 8} p(x, t) d x+(\delta(a))^{2}\right) \cdot\|\psi\|^{2},
\end{aligned}
$$

and this tends to zero as $a$ becomes infinite, uniformly for $\psi$ in $L^{2}(\bar{\Omega})$ with $\|\psi\|=1$. The proof of Tamarkin's criterion, and hence of the complete continuity of $K_{t}$ in the cases stated, is now complete.

That the complete continuity of $K_{t}$, together with the fact that $K_{t}$ is positive definite, imply that $K_{t}$ has a sequence of eigenvalues from whose 
eigenfunctions a complete orthonormal basis may be chosen is well known.

The remainder of the proof is also quite standard. If $\phi$ is an eigenfunction of $K_{t}$ with eigenvalue $\mu$, we have clearly $0<\mu<1$ since $K_{t}$ is positive definite and a strict contraction; set $\lambda=-(1 / t) \log \mu . \phi$ is also an eigenfunction of $K_{t / 2}$ with eigenvalue $\exp \{-\lambda(t / 2)\}=\mu^{1 / 2}$. For $\left(K_{t}-\mu I\right) \phi=\left(K_{t / 2}+\mu^{1 / 2} I\right)\left(K_{t / 2}-\mu^{1 / 2} I\right) \phi=0$, but setting $\psi=K_{t / 2} \phi-\mu^{1 / 2} \phi, \quad 0=\left\|\left(K_{t / 2}+\mu^{1 / 2} I\right) \psi\right\|^{2}=\left(\left(K_{t / 2}+\mu^{1 / 2} I\right)^{2} \psi, \quad \psi\right)$ $=\left\|K_{t} \psi\right\|^{2}+\mu^{2}\|\psi\|^{2}+2 \mu^{1 / 2}\left(K_{t / 2} \psi, \psi\right)$, so that $\psi=K_{t / 2} \phi-\mu^{1 / 2} \phi=0$ follows from the fact that $K_{t / 2}$ is positive definite. Repeating the process, we see that $\phi$ is an eigenfunction of $K_{t}$, with eigenvalue $\exp \left\{-\lambda t^{\prime}\right\}$, whenever $t^{\prime}=\left(m / 2^{n}\right) t$ for positive integers $m, n$. Since such numbers are dense in the half line $\left\{t^{\prime}>0\right\}$, the strong continuity of the semi-group implies that $\phi$ is an eigenfunction of $K_{t}$, with eigenvalue $e^{-\lambda t}$, for every $t^{\prime}>0$.

We use the relation between the semi-group and the operator $L$, and use Lemma 3 to prove the first of the results stated in the introduction.

Theorem III. Let $\Omega$ and $V(x)$ be as in Theorem I, and suppose also either that $\Omega$ is bounded, or that $\lim _{|x| \rightarrow \infty, x \in \Omega} V(x)=\infty$. Then the differential operator $L \equiv \Delta / 2-V(x)$ on $L^{2}(\bar{\Omega})$, with boundary values zero, has a discrete spectrum of negative eigenvalues $\left\{-\lambda_{n}\right\}$, with eigenfunctions forming a complete orthonormal basis in $L^{2}(\bar{\Omega})$.

Theorem III is a direct consequence of Lemma 3 and the fact that $\phi(x)$ is an eigenfunction of $L$ with eigenvalue $-\lambda$ if and only if $\phi(x)$ is an eigenfunction of $K_{t}$ for each $t>0$, with eigenvalue $e^{-\lambda t}$. These facts are quite simple, however: If $\phi$ in $L^{2}(\bar{\Omega})$ and $\lambda$ are such that $K_{t} \phi=e^{-\lambda t} \phi$ for $t>0$, then by Theorem I,

$$
\begin{aligned}
L(\exp \{-\lambda t\} \phi) & \equiv \exp \{-\lambda t\}\left(\frac{1}{2} \Delta \phi-V(x) \phi\right) \\
& =\frac{\partial}{\partial t}(\exp \{-\lambda t\} \phi)=-\lambda \exp \{-\lambda t\} \phi
\end{aligned}
$$

so that $\phi$ is an eigenfunction of $L$ with eigenvalue $-\lambda$. On the other hand, if $-\lambda$ is an eigenvalue of $L$, with eigenfunction $\phi$ in $L^{2}(\bar{\Omega})$, then by Theorem I,

$$
\begin{aligned}
H_{s} \phi(x) & =\int_{\bar{\Omega}} H(x, y ; s) \phi(y) d y=\int_{0}^{\infty} e^{-s t} K_{t} \phi(x) d t \\
& =\frac{1}{s+\lambda} \phi(x)=\int_{0}^{\infty} e^{-s t} e^{-\lambda t} \phi(x) d t
\end{aligned}
$$

for every $s>0$. By the uniqueness of the Laplace transforms $K_{t} \phi$ $=\exp \{-\lambda t\} \phi$ for all $t>0$, which completes the proof.

4. Asymptotic behavior of the eigenfunctions and eigenvalues of $L$. In this last section of the paper we give the asymptotic relations stated in the 
introduction for the eigenfunctions and eigenvalues of $L$. The first three of these results are now very easily proved, and serve to illustrate the method used.

TheOREM IV. Let $\Omega$ and $V(x)$ be as in Theorem I, and suppose also either that $\Omega$ is bounded, or that $\lim _{|x| \rightarrow \infty, x \in \Omega} V(x)=\infty$. Then the normalized eigenfunctions $\left\{\phi_{n}(x)\right\}$ of the differential operator $L \equiv 2^{-1} \Delta-V(x)$, with boundary values zero on $\Omega$, satisfy

$$
\sum_{\lambda_{n}<\lambda} \phi_{n}^{2}(x) \sim\left(\frac{\lambda}{2 \pi}\right)^{N / 2} \frac{1}{\Gamma(N / 2+1)}
$$

as $\lambda \rightarrow \infty$, for each $x$ in $\Omega$.

Since each eigenfunction $\phi_{n}$ of $L$ is also an eigenfunction of $K_{t}$, with eigenvalue $\exp \left\{-\lambda_{n} t\right\}$, and since $\left\{\phi_{n}\right\}$ is an orthonormal basis in $L^{2}(\bar{\Omega})$, it is easy to verify that

$$
K(x, y ; t)=\sum_{n} \exp \left\{-\lambda_{n} t\right\} \phi_{n}(x) \phi_{n}(y)
$$

for $x, y$ in $\Omega$ and $t>0$. Hence

$$
\begin{aligned}
\sum_{n} \exp \left\{-\lambda_{n} t\right\} \phi_{n}^{2}(x)= & K(x, x ; t) \\
= & \frac{1}{(2 \pi t)^{N / 2}} E\left\{\exp \left\{-\int_{0}^{t} V(x+x(\tau)) d \tau\right\} ;\right. \\
& x+x(\tau) \in \bar{\Omega}, 0 \leqq \tau \leqq t \mid x(t)=0\} \\
= & \int_{0}^{\infty} \exp \{-\lambda t\} d_{\lambda}\left(\sum_{\lambda_{n}<\lambda} \phi_{n}^{2}(x)\right) .
\end{aligned}
$$

Now as $t \rightarrow 0$, it is clear that for $x$ in $\Omega$,

$$
E\left\{\exp \left\{-\int_{0}^{t} V(x+x(\tau)) d \tau\right\} ; x+x(\tau) \in \bar{\Omega}, 0 \leqq \tau \leqq t \mid x(t)=0\right\}
$$

tends to 1 . For

$$
\exp \left\{-\int_{0}^{t} V(x+x(\tau)) d \tau\right\} \rightarrow 1
$$

for each of the continuous paths $x(\cdot)$ of the Wiener process, while by Lemma 2 if $x \in \Omega$,

$$
\operatorname{Prob}\{x+x(\tau) \in \bar{\Omega}, 0 \leqq \tau \leqq t \mid x(t)=0\} \rightarrow 1
$$


Therefore

$$
K(x, x ; t) \sim \frac{1}{(2 \pi t)^{N / 2}}
$$

as $t \rightarrow 0$,

for each $x$ in $\Omega$. Applying the Hardy-Littlewood-Karamata Tauberian Theorem $[10$, p. 208$]$, this and equation (11) imply

$$
\sum_{\lambda_{n}<\lambda} \phi_{n}^{2}(x) \sim\left(\frac{\lambda}{2 \pi}\right)^{N / 2} \frac{1}{\Gamma(N / 2+1)} \quad \text { as } \lambda \rightarrow \infty .
$$

Theorem V. Let $\Omega$ and $V(x)$ be as in Theorem I, and suppose that $\Omega$ is bounded. Then the eigenvalues $\left\{-\lambda_{n}\right\}$ of the differential operator $L \equiv 2^{-1} \Delta-V(x)$ with boundary value zero on $\Omega$ satisfy

$$
N(\lambda) \equiv \sum_{\lambda_{n}<\lambda} 1 \sim\left(\frac{\lambda}{2 \pi}\right)^{N / 2} \frac{m \Omega}{\Gamma(N / 2+1)} \quad \text { as } \lambda \rightarrow \infty .
$$

By equation (11), the Laplace-Stieltjes transform of

$$
N(\lambda)=\sum_{\lambda_{n}<\lambda} \int_{\Omega} \phi_{n}^{2}(x) d x
$$

is

$$
\int_{\mathbf{a}} K(x, x ; t) d x .
$$

As in the proof of Theorem IV, $(2 \pi t)^{N / 2} K(x, x ; t) \rightarrow 1$ and $t \rightarrow 0$, and the convergence is bounded, so that

$$
\int_{\Omega} K(x, x ; t) d x \sim \frac{m \Omega}{(2 \pi t)^{N / 2}} \quad \text { as } t \rightarrow 0 .
$$

Again, the Karamata Tauberian Theorem implies

$$
N(\lambda) \sim\left(\frac{\lambda}{2 \pi}\right)^{N / 2} \frac{m \Omega}{\Gamma(N / 2+1)}, \quad \text { as } \lambda \rightarrow \infty .
$$

To extend the result of Theorem $V$ to the case of $\Omega$ unbounded requires more delicate treatment, and can be carried out only if the asymptotic behavior of $V(x)$ as $|x| \rightarrow \infty$ is assumed such that the Karamata Tauberian Theorem is applicable. In addition, a smoothness condition must be satisfied by $V(x)$; although this is very weak, we cannot show that the asymptotic behavior of $N(\lambda)$ depends only on the asymptotic behavior of $V(x)$.

Theorem VI. Let $\Omega$ and $V(x)$ be as in Theorem I, and assume that $\Omega$ is unbounded and that $\lim _{|x| \rightarrow \infty, x \in \mathbb{\Omega}} V(x)=\infty$. Suppose also that 


$$
m\{x \in \bar{\Omega} \mid V(x)<\lambda\} \sim \lambda^{\alpha} F(\lambda),
$$

$$
\alpha \geqq 0,
$$

with $F(\lambda)$ slowly increasing: that is, for every $\mu>0$,

$$
\lim _{\lambda \rightarrow \infty} \frac{F(\mu \lambda)}{F(\lambda)}=1 \text {; }
$$

and that for some $\delta>0$,

$$
m\{x \in \bar{\Omega} \mid \underset{|y|<\delta, x+y \in \overline{\mathbf{\Omega}}}{\operatorname{Max}} V(x+y)<\lambda\} \sim \lambda^{\alpha} F(\lambda) .
$$

Then the eigenvalues $\left\{-\lambda_{n}\right\}$ of the differential operator $L \equiv 2^{-1} \Delta-V(x)$ with boundary values zero on $\Omega$ satisfy

$$
\begin{aligned}
N(\lambda) & \equiv \sum_{\lambda_{n}<\lambda} 1 \sim \frac{1}{(2 \pi)^{N}} B(\lambda) \\
& =\frac{1}{(2 \pi)^{N}} m\left\{x,\left.y\left|\frac{1}{2}\right| y\right|^{2}+V(x)<\lambda, x \in \bar{\Omega}\right\},
\end{aligned}
$$

as $\lambda \rightarrow \infty$.

We start the proof of Theorem VI from the relation

$$
\int_{0}^{\infty} \exp \{-\lambda t\} d N(\lambda)=\int_{\bar{\Omega}} K(x, x ; t) d x
$$

derived from equation (11). By the Karamata Tauberian theorem, $N(\lambda)$ $\sim\left(1 /(2 \pi)^{N}\right) B(\lambda)$ will follow if we can prove

$$
\int_{\bar{\Omega}} K(x, x ; t) d x \sim \frac{1}{(2 \pi t)^{N / 2}} \int_{\bar{\Omega}} \exp \{-t V(x)\} d x .
$$

For since $m\{x \in \bar{\Omega} \mid V(x)<\lambda\} \sim \lambda^{\alpha} F(\lambda)$, an Abelian argument [10, p. 201] implies

$$
\begin{aligned}
\frac{1}{(2 \pi t)^{N / 2}} \int_{\bar{\Omega}} \exp \{-t V(x)\} d x & =\frac{1}{(2 \pi t)^{N / 2}} \int_{0}^{\infty} e^{-\lambda t} d m\{V(x)<\lambda\} \\
& \sim \frac{1}{(2 \pi t)^{N / 2}} \Gamma(\alpha+1) F\left(\frac{1}{t}\right) t^{-\alpha}
\end{aligned}
$$

Hence the Tauberian Theorem yields

$$
N(\lambda) \sim \frac{\Gamma(\alpha+1)}{\Gamma(\alpha+N / 2+1)} \frac{F(\lambda)}{(2 \pi)^{N / 2}} \lambda^{N / 2+\alpha} .
$$

But it is easy to see that 


$$
\begin{aligned}
\frac{1}{(2 \pi t)^{N / 2}} \int_{\bar{\Omega}} \exp \{-t V(x)\} d x \\
=\frac{1}{(2 \pi)^{N}} \int_{R^{N}} \exp \left\{-t \frac{|y|^{2}}{2}\right\} d y \int_{\bar{\Omega}} \exp \{-t V(x)\} d x \\
=\frac{1}{(2 \pi)^{N}} \int_{0}^{\infty} \exp \{-\lambda t\} d B(\lambda),
\end{aligned}
$$

whence by the same argument,

$$
N(\lambda) \sim \frac{1}{(2 \pi)^{N}} B(\lambda) \sim \frac{\Gamma(\alpha+1)}{\Gamma(\alpha+1+N / 2)} \frac{F(\lambda)}{(2 \pi)^{N / 2}} \lambda^{N / 2+\alpha} .
$$

Thus the proof of Theorem VI is reduced to that of equation (12).

We prove equation (12) in two steps, the first of which is relatively simple:

$$
\begin{aligned}
\int_{\bar{\Omega}} K(x, x ; t) d x & =\int_{\bar{\Omega}} d x \frac{1}{(2 \pi t)^{N / 2}} E\left\{\exp \left\{-\int_{0}^{t} V(x+x(\tau)) d \tau\right\} ;\right. \\
& x+x(\tau) \in \bar{\Omega}, 0 \leqq \tau \leqq t \mid x(t)=0\} \\
\leqq & \int_{\bar{\Omega}} d x \frac{1}{(2 \pi t)^{N / 2}} \frac{1}{t} \int_{0}^{t} d \tau \\
& \cdot E\{\exp \{-t V(x+x(\tau))\} ; x+x(\tau) \in \bar{\Omega}, 0 \leqq \tau \leqq t \mid x(t)=0\}
\end{aligned}
$$

by Jensen's inequality,

$$
\begin{aligned}
& =\frac{1}{(2 \pi t)^{N / 2}} \frac{1}{t} \int_{0}^{t} d \tau E\left\{\int_{x \in \bar{\Omega}, x+x(\tau) \in \bar{\Omega}} \exp \{-t V(x+x(\tau))\} d x \mid x(t)=0\right\} \\
& \leqq \frac{1}{(2 \pi t)^{N / 2}} \frac{1}{t} \int_{0}^{t} d \tau E\left\{\int_{\bar{\Omega}} \exp \{-t V(x)\} d x \mid x(t)=0\right\} \\
& =\frac{1}{(2 \pi t)^{N / 2}} \int_{\Omega} \exp \{-t V(x)\} d x .
\end{aligned}
$$

Therefore

$$
\int_{\bar{\Omega}} K(x, x ; t) d x \leqq \frac{1}{(2 \pi t)^{N / 2}} \int_{\bar{\Omega}} \exp \{-t V(x)\} d x, \quad t>0 .
$$
first

To prove that asymptotically, the opposite inequality holds, we write

$$
f(u, v)=0 \quad \text { if } u \leqq v, \quad f(u, v)=1 \quad \text { if } u>v .
$$

Then 


$$
\begin{aligned}
& \int_{\bar{\Omega}} K(x, x ; t) d x \\
& =\frac{1}{(2 \pi t)^{N / 2}} \int_{\bar{\Omega}} d x E\left\{\int_{0}^{\infty} \exp \{-u\} f\left(u, \int_{0}^{t} V(x+x(\tau)) d \tau\right) d u\right. \\
& x+x(\tau) \in \bar{\Omega}, 0 \leqq \tau \leqq t \mid x(t)=0\} \\
& =\frac{1}{(2 \pi t)^{N / 2}} \int_{0}^{\infty} \exp \{-u\} d u \\
& \cdot E\left\{\int_{x \in \bar{\Omega}, x+x(\tau) \in \bar{\Omega}, 0 \leqq \tau \leqq t} f\left(u, \int_{0}^{t} V(x+x(\tau)) d \tau\right) d x \mid x(t)=0\right\} \\
& =\frac{1}{(2 \pi t)^{N / 2}} \int_{0}^{\infty} \exp \{-u\} d u \\
& \cdot E\left\{m \left\{x \in \bar{\Omega} \mid \int_{0}^{t} V(x+x(\tau)) d \tau<u ;\right.\right. \\
& x+x(\tau) \in \bar{\Omega}, 0 \leqq \tau \leqq t\} \mid x(t)=0\} \\
& \geqq \frac{1}{(2 \pi t)^{N / 2}} \int_{0}^{\infty} \exp \{-u\} d u \\
& \cdot E\left\{m\left\{x \in \bar{\Omega} \mid \int_{0}^{t} V(x+x(\tau)) d \tau<u, x+x(\tau) \in \bar{\Omega}, 0 \leqq \tau \leqq t\right\} ;\right. \\
& \underset{0 \leqq \tau \leqq t}{\operatorname{Max}}|x(\tau)|<\delta \mid x(t)=0\} \text {, }
\end{aligned}
$$

since the Wiener integral over the complementary subset $\left\{\operatorname{Max}_{0 \leqq \tau \leqq t}|x(\tau)|\right.$ $\geqq \delta\}$ of $C_{t, 0}$ is certainly non-negative. But if $\operatorname{Max}_{0 \leqq \tau \leqq t}|x(\tau)|<\delta$, then $t \operatorname{Max}_{|y|<\delta} V(x+y)<u$ implies $\int_{0}^{t} V(x+x(\tau)) d \tau<u$, so that

$$
\begin{aligned}
& \int_{\Omega} K(x, x ; t) d x \geqq \frac{1}{(2 \pi t)^{N / 2}} \int_{0}^{\infty} \exp \{-u\} d u \\
& \cdot E\{m\{x \in \bar{\Omega} \mid t \underset{|y|<\delta, x+y \in \bar{\Omega}}{\operatorname{Max}} V(x+y)<u\} ; \underset{0 \leqq \tau \leqq t}{\operatorname{Max}}|x(\tau)|<\delta \mid x(t)=0\} \\
& \geqq \frac{1}{(2 \pi t)^{N / 2}} \int_{0}^{\infty} e^{-u} d u \cdot m\left\{x \in \bar{\Omega} \mid \underset{|y|<\delta, x+y \in \bar{\Omega}}{\operatorname{Max}} V(x+y)<\frac{u}{t}\right\} \\
& \cdot\left(1-3 \int_{|y|>\delta / 4} p(y, t) d y\right) .
\end{aligned}
$$


Now as $t \rightarrow 0,1-3 \int_{|y|>\delta / 4} p(y, t) d y \rightarrow 1$, while by the Abelian argument presented before, and by the hypothesis

$$
m\left\{x \in \bar{\Omega} \mid \operatorname{Max}_{|y|<\delta, x+\nu \in \bar{\Omega}} V(x)<\lambda\right\} \sim \lambda^{\alpha} F(\lambda),
$$

we infer

$$
\int_{0}^{\infty} \exp \{-u\} d u m\left\{x \in \bar{\Omega} \mid \operatorname{Max}_{|y|<\delta, x+\nu \in \bar{\Omega}} V(x)<\frac{u}{t}\right\} \sim \Gamma(\alpha+1) F\left(\frac{1}{t}\right) t^{-\alpha} .
$$

From these considerations, together with (13), it follows that

$$
\begin{aligned}
\int_{\bar{\Omega}} K(x, x ; t) d x & \sim \frac{1}{(2 \pi t)^{N / 2}} \int_{\bar{\Omega}} \exp \{-t V(x)\} d x \\
& \sim \frac{1}{(2 \pi t)^{N / 2}} \Gamma(\alpha+1) F\left(\frac{1}{t}\right) t^{-\alpha}
\end{aligned}
$$

as $t \rightarrow 0$. This proves equation (12), thus completing the proof of Theorem VI.

In order to illustrate the dependence of the result of Theorem VI on the hypothesis, we consider the potential $V(x)=\log ^{+}|x|=\operatorname{Max}\{\log |x|, 0\}$ on $\Omega=R^{N}$. None of the hypotheses concerning the asymptotic behavior of $V(x)$ are satisfied for this potential; still, some partial information can be deduced concerning the eigenvalues of $L=\Delta / 2-\log ^{+}|x|$.

By Theorem III, $L=2^{-1} \Delta-\log ^{+}|x|$ has a discrete spectrum of negative eigenvalues $\left\{-\lambda_{n}\right\}$ with eigenfunctions $\left\{\phi_{n}(x)\right\}$, satisfying

$$
\begin{aligned}
& \sum_{n} \exp \left\{-\lambda_{n} t\right\} \phi_{n}^{2}(x) \\
& \quad=K(x, x ; t)=\frac{1}{(2 \pi t)^{N / 2}} E\left\{\exp \left\{-\int_{0}^{t} \log ^{+}|x+x(\tau)| d \tau\right\} \mid x(t)=0\right\} .
\end{aligned}
$$

Moreover if $t>N$

$$
\begin{aligned}
& \sum_{n} \exp \left\{-\lambda_{n} t\right\}=\int_{0}^{\infty} \exp \{-\lambda t\} d N(\lambda) \\
& =\frac{1}{(2 \pi t)^{N / 2}} \int_{R^{N}} d x E\left\{\exp \left\{-\int_{0}^{t} \log ^{+}|x+x(\tau)| d \tau\right\} \mid x(t)=0\right\}
\end{aligned}
$$

but both sides of the equation are infinite if $t \leqq N$. We can still apply Karamata's Theorem, however, to the relation

$$
\int_{0}^{\infty} \exp \{-\lambda t\} \exp \{-\lambda N\} d N(\lambda)=\int_{R^{N}} K(x, x ; t+N) d x .
$$


As in the proof of Theorem VI,

$$
\begin{aligned}
\frac{2 \pi^{N / 2}}{\Gamma(N / 2)}\left(\frac{1}{N}+\frac{1}{t}\right)=\int_{R^{N}} \exp \left\{-(t+N) \log ^{+}|x|\right\} d x \\
\geqq(2 \pi(t+N))^{N / 2} \int_{R^{N}} K(x, x ; t+N) d x \\
\geqq \int_{0}^{\infty} \exp \{-u\} d u m\left\{x \in R^{N}\left|\operatorname{Max}_{|y|<\delta} \log ^{+}\right| x+y \mid<\frac{u}{t+N}\right\} \\
\\
\cdot\left(1-3 \int_{|y|>\delta / 4} p(y, t) d y\right) \\
=\frac{\pi^{N / 2}}{\Gamma(N / 2+1)}(t+N)\left(\sum_{0}^{N} \frac{N !}{k !(N-k) !} \frac{\delta^{k}}{t+k}\right) \\
\cdot\left(1-3 \int_{|y|>\delta / 4} p(y, t) d y\right) .
\end{aligned}
$$

Hence as $t \rightarrow 0$,

$$
\int_{R^{N}} K(x, x ; t+N) d x \sim \frac{1}{(2 N)^{N / 2}} \frac{1}{\Gamma(N / 2)} \frac{2}{t}
$$

applying Karamata's Tauberian theorem,

$$
\int_{0}^{\lambda} \exp \{-N \mu\} d N(\mu)=\sum_{\lambda_{n}<\lambda} \exp \left\{-N \lambda_{n}\right\} \backsim \frac{1}{(2 N)^{N / 2}} \frac{1}{\Gamma(N / 2)} 2 \lambda .
$$

On the average, therefore, the number $N(\lambda)$ of eigenvalues of the potential $\log ^{+}|x|$ behaves like $2(2 N)^{-N / 2} / \Gamma(N / 2) \cdot e^{N \lambda}$, although it seems impossible to prove asymptotic equality of $N(\lambda)$ with this expression without using more than just the asymptotic behavior of the potential $V(x)$.

Added in proof. The author has learned that using Tauberian arguments, Professor Titchmarsh has recently derived asymptotic relations for the eigenvalues of the differential operator $L$ in dimensions greater than one. His new results bear much the same relationship to ours as do his previous results in the one-dimensional case.

\section{REFERENCES}

1. M. Kac, On some connections between probability theory and differential and integral equations, Proceedings of the Second Berkeley Symposium on Mathematical Statistics and Probability, University of California Press, 1951, pp. 189-215.

2. M. Rosenblatt, On a class of Markov processes, Trans. Amer. Math. Soc. vol. 71 (1951) pp. 120-135.

3. E. C. Titchmarsh, Eigenfunction expansions associated with second-order differential equations, Oxford, 1946. 
4. F. G. Dressel, The fundamental solution of the parabolic equation, Duke Math. J. vol. 7 (1940) pp. 186-203.

5. R. E. A. C. Paley and N. Wiener, Fourier transforms in the complex domain, Amer. Math. Soc. Colloquium Publications, vol. 19, 1934.

6. P. Levy, Le mouvement Brownien plan, Amer. J. Math. vol. 62 (1940) pp. 487-550.

7. J. D. Tamarkin, On the compactness of the space $L_{p}$, Bull. Amer. Math. Soc. vol. 38 (1932) pp. 79-84.

8. A. N. Kolmogorov, Foundations of the theory of probability, Chelsea, 1950.

9. R. Courant and D. Hilbert, Methoden der mathematischen Physik, vol. I, Springer, 1931.

10. G. Doetsch, Theorie und Anwendung der Laplace-Transformation, Dover, 1943.

\author{
National Bureau of Standards, \\ Los ANgeles, Calif. \\ CoRnell University, \\ ITHACA, N. Y.
}

